\title{
Markov Dilations and Quantum Detailed Balance
}

\author{
Alberto Frigerio and Vittorio Gorini \\ Dipartimento di Fisica, Sezione di Fisica Teorica, Università di Milano, Milano, Italy
}

\begin{abstract}
We construct quantum stochastic processes whose multi-time correlation functions, with suitable time ordering, can be obtained from a quantum dynamical semigroup. We prove that such a process defines a stationary Markov dilation of the associated semigroup if and only if (up to technicalities) the semigroup satisfies the quantum detailed balance condition with respect to its stationary state.
\end{abstract}

\section{Introduction}

Quantum dynamical semigroups have been widely used in the last ten years to describe irreversible time evolutions of open systems. The connection with the underlying Hamiltonian dynamics of isolated systems has been investigated in both directions. For a class of models of open systems, it has been proved that the reduced evolution converges to a dynamical semigroup in the weak or the singular coupling limit $[1,2]$. Conversely, it has been shown that any dynamical semigroup $\Phi_{t}$ on a $C^{*}$-algebra $\mathscr{B}$ admits a unitary dilation, consisting of an embedding $j_{0}$ of $\mathscr{B}$ into another $C^{*}$-algebra $\mathscr{A}$, a group $\alpha_{t}$ of $*$-automorphisms of $\mathscr{A}$ and a norm one projection $E_{0}$ of $\mathscr{A}$ onto $j_{0}(\mathscr{B})$ such that $\Phi_{t}=j_{0}^{-1} E_{0} \alpha_{t} j_{0}$ for all $t$ in $\mathbb{R}^{+}$[3-5]; however, the unitary dilation is far from unique.

More recently, it was recognized that a tighter connection between irreversible evolution and underlying Hamiltonian dynamics could be obtained by the consideration of multi-time correlation functions [6]. This leads to the idea of a quantum stochastic process [7-9], which should be determined up to equivalence by its multi-time correlations, much in the same way as a stochastic process in the sense of Doob is determined by its finite-dimensional joint distributions. Then it becomes possible to require that the unitary dilation defines a Markov process. The convergence of multi-time correlations in the weak or the singular coupling limit was proved by Dümcke $[10,11]$ for a class of models; the converse problem of Markov dilations has been investigated by Kümmerer and Schröder [12-14, 11]. Related works include the generalized $K$-flows of Emch et al. $[15,16]$ and the 
construction of quantum stochastic processes via the solution of noncommutative stochastic differential equations, given by Hudson and Parthasarathy $[17,11]$, see also von Waldenfels $[18,11]$.

In the present paper, we associate a quantum stochastic process (in the sense of Accardi et al. [8]) with a quantum dynamical semigroup of Lindblad type [19]. The construction is made via Dümcke's results on the convergence of multi-time correlations in the singular coupling limit [10] and the reconstruction theorem of [8]. Next, we prove that the process is stationary and Markov if and only if (up to minor technicalities) the dynamical semigroup satisfies the quantum detailed balance condition $[20,21]$ with respect to its stationary state.

Section 2 of the paper contains a collection of the definitions we need. We give the construction of the process in Sect. 3, and we prove the connection with quantum detailed balance in Sect. 4.

Before entering the technical details, we wish to give a rough idea of what is going on. A quantum stochastic process (in the sense of [8]) over a $C^{*}$ - or $W^{*}$ algebra $\mathscr{B}$ consists of a family $\left\{j_{t}: t \in \mathbb{R}\right\}$ of identity preserving embeddings of $\mathscr{B}$ into another $C^{*}$ - or $W^{*}$-algebra $\mathscr{A}$, together with a state $\mu$ on $\mathscr{A}$. It defines a unitary dilation of a dynamical semigroup $\Phi_{t}$ on $\mathscr{B}$ if, in addition, there exists a group $\left\{\alpha_{t}: t \in \mathbb{R}\right\}$ of $*$-automorphisms of $\mathscr{A}$ such that $j_{t}=\alpha_{t} j_{0}$, and the state $\mu$ is obtained as $\mu=\varrho \circ j_{0}^{-1} E_{0}, \varrho$ being a state on $\mathscr{B}$, and $E_{0}$ being a norm one projection onto $j_{0}(\mathscr{B})$ such that $\Phi_{t}=j_{0}^{-1} E_{0} \alpha_{t} j_{0}$. Conversely, a unitary dilation of $\Phi_{t}$ and a state $\varrho$ on $\mathscr{B}$ define a quantum stochastic process.

A process can be reconstructed uniquely up to equivalence from its correlation kernels [8], defined as

$$
w_{t_{1}, \ldots, t_{n}}\left(A_{1}, \ldots, A_{n} ; B_{1}, \ldots, B_{n}\right)=\mu\left(j_{t_{1}}\left(A_{1}^{*}\right) \ldots j_{t_{n}}\left(A_{n}^{*}\right) j_{t_{n}}\left(B_{n}\right) \ldots j_{t_{1}}\left(B_{1}\right)\right),
$$

$A_{1}, \ldots, A_{n}, B_{1}, \ldots, B_{n} \in \mathscr{B}, t_{1}, \ldots, t_{n} \in \mathbb{R}, n \in \mathbb{N}$. However, a dynamical semigroup $\Phi_{t}$ and a state $\varrho$ on $\mathscr{B}$ can only determine the time-ordered correlation kernels $\left(0 \leqq t_{1} \leqq t_{2} \leqq \ldots \leqq t_{n}\right)$ via

$$
w_{t_{1}, \ldots, t_{n}}\left(A_{1}, \ldots, A_{n} ; B_{1}, \ldots, B_{n}\right)=\varrho\left(\Phi_{t_{1}}\left(A_{1}^{*} \Phi_{t_{2}-t_{1}}\left(A_{2}^{*} \ldots \Phi_{t_{n}-t_{n-1}}\left(A_{n}^{*} B_{n}\right) \ldots B_{2}\right) B_{1}\right)\right) .
$$

The time-ordered kernels (1.2) do not suffice to determine all the correlation kernels (1.1), unless the commutation relations of $\mathscr{A}$ are known in advance, as is the case for classical systems, or for quasi-free Bose or Fermi systems [16, 22, 23, 8]. In the general situation, a dynamical semigroup may have inequivalent unitary dilations satisfying (1.2), corresponding, for instance, to an interaction of the system of interest with a reservoir of boson or of fermion type.

What is worse, Eq. (1.2) does not provide sufficient information for the construction of a quantum stochastic process associated with a dynamical semigroup. Hence we must resort to a different method of construction; here we employ the results of Dümcke [10] to define the correlation kernels of the process as

$$
\begin{aligned}
& w_{t_{1}, \ldots, t_{n}}\left(A_{1}, \ldots, A_{n} ; B_{1}, \ldots, B_{n}\right) \\
& \quad=\lim _{\varepsilon \rightarrow 0} \varrho \otimes \varrho^{R}\left(\alpha_{t_{1}}^{\varepsilon}\left(A_{1}^{*} \otimes \mathbb{1}\right) \ldots \alpha_{t_{n}}^{\varepsilon}\left(A_{n}^{*} \otimes \mathbb{1}\right) \alpha_{t_{n}}^{\varepsilon}\left(B_{n} \otimes \mathbb{1}\right) \ldots \alpha_{t_{1}}^{\varepsilon}\left(B_{1} \otimes \mathbb{1}\right)\right),
\end{aligned}
$$


where $\left\{\alpha_{t}^{\varepsilon}: t \in \mathbb{R}\right\}$ is the time evolution of a composite system, becoming more and more singular as $\varepsilon \rightarrow 0$. The process constructed in this way satisfies (1.2). We do not know, in general, whether the limiting process $\left(\mathscr{A},\left\{j_{t}: t \in \mathbb{R}\right\}, \mu\right)$ is equipped with a group $\alpha_{t}$ of $*$-automorphisms of $\mathscr{A}$ such that $j_{t}=\alpha_{t} j_{0}$. However, this is certainly the case if the process is stationary: then also $\mu \circ \alpha_{t}=\mu$ and $\varrho \circ \Phi_{t}=\varrho$. Moreover, if we let $\Phi_{t}^{+}=j_{0}^{-1} E_{0} \alpha_{-t} j_{0}, t \in \mathbb{R}^{+}$, we have

$$
\varrho\left(\Phi_{t}^{+}(A) B\right)=\varrho\left(A \Phi_{t}(B)\right) \text { for all } A, B \text { in } \mathscr{B}, t \text { in } \mathbb{R}^{+},
$$

and it follows from the construction (cf. [24]) that the infinitesimal generators $L$ and $L^{+}$of $\Phi_{t}$ and $\Phi_{t}^{+}$respectively satisfy

$$
L(B)-L^{+}(B)=2 i[H, B] \quad\left(H=H^{*}\right) \quad \text { for all } B \text { in } \mathscr{B} .
$$

Equations (1.4) and (1.5) together define the quantum detailed balance condition $[20,21]$. Conversely, for a quantum dynamical semigroup of Lindblad type satisfying the detailed balance condition, we prove that the associated process is stationary. The Markov property, defined in terms of conditional expectations, follows with the aid of Takesaki's theorem [25].

As pointed out by Kümmerer [12], Eq. (1.4) is a necessary condition for the existence of a stationary Markov dilation. The additional condition (1.5) seems to be related to the method of construction; however, we are not aware of any example of a stationary Markov dilation for a quantum dynamical semigroup which does not satisfy detailed balance.

\section{Definitions}

Throughout the following, $\mathscr{B}$ will denote a $W^{*}$-algebra.

A dynamical semigroup $\left\{\Phi_{t}: t \in \mathbb{R}^{+}\right\}$on $\mathscr{B}$ is a weakly * continuous semigroup of completely positive identity preserving normal linear maps of $\mathscr{B}$ into itself, $\Phi_{0}$ being the identity map.

A dynamical semigroup $\Phi_{t}$ on $\mathscr{B}$ will be said to be of finite Lindblad type if it is norm continuous, with infinitesimal generator $L$ given by

$$
L(B)=i[H, B]+\sum_{j=1}^{N} V_{j}^{*} B V_{j}-\frac{1}{2}\left[V_{j}^{*} V_{j}, B\right]_{+}
$$

for all $B$ in $\mathscr{B}$, where $H=H^{*} \in \mathscr{B}, V_{j} \in \mathscr{B}, j=1, \ldots, N$ (cf. [19]).

A norm continuous dynamical semigroup $\Phi_{t}=\exp L t$ on $\mathscr{B}$ is said to satisfy the detailed balance condition [20,21] with respect to a faithful normal state $\varrho$ on $\mathscr{B}$ if there exists another norm continuous dynamical semigroup $\Phi_{t}^{+}=\exp L^{+} t$ on $\mathscr{B}$ such that

$$
\varrho\left(L^{+}(A) B\right)=\varrho(A L(B)) \text { for all } A, B \text { in } \mathscr{B},
$$

and

$$
L(B)-L^{+}(B)=2 i[H, B], H=H^{*} \in \mathscr{B}, \quad \text { for all } B \text { in } \mathscr{B} .
$$

A stochastic process $[8,9]$ over $\mathscr{B}$ is a triple $\left(\mathscr{A},\left\{j_{t}: t \in \mathbb{R}\right\}, \mu\right)$, where $\mathscr{A}$ is a $C^{*}$ algebra, $j_{t}$ is a ${ }^{*}$-representation of $\mathscr{B}$ into $\mathscr{A}$, with $j_{t}\left(\mathbb{1}_{\mathscr{B}}\right)=\mathbb{1}_{\mathscr{A}}$, for each $t$ in $\mathbb{R}, \mathscr{A}$ is generated by $j_{t}(\mathscr{B}): t \in \mathbb{R}$, and $\mu$ is a state on $\mathscr{A}$. 
Two stochastic processes over $\mathscr{B}$ are said to be equivalent if they have the same correlation kernels, defined by

$$
w_{t_{1}, \ldots, t_{n}}\left(A_{1}, \ldots, A_{n} ; B_{1}, \ldots, B_{n}\right)=\mu\left(j_{t_{1}}\left(A_{1}^{*}\right) \ldots j_{t_{n}}\left(A_{n}^{*}\right) j_{t_{n}}\left(B_{n}\right) \ldots j_{t_{1}}\left(B_{1}\right)\right),
$$

for all $A_{1}, \ldots, A_{n}, B_{1}, \ldots, B_{n}$ in $\mathscr{B}, t_{1}, \ldots, t_{n}$ in $\mathbb{R}$, and $n$ in $\mathbb{N}$. By going to the GNS representation and taking the double commutant, it is always possible (up to equivalence) to assume that $\mathscr{A}$ is a $W^{*}$-algebra and $\mu$ is a normal state; we shall do so in the following. In the special case when $j_{t}$ is faithful and normal for all $t$, we shall say that $\left(\mathscr{A},\left\{j_{t}: t \in \mathbb{R}\right\}, \mu\right)$ is a $W^{*}$-stochastic process.

A stochastic process is said to be stationary if, for all $A_{1}, \ldots, A_{n}, B_{1}, \ldots, B_{n}$ in $\mathscr{B}, t_{1}, \ldots, t_{n}, t$ in $\mathbb{R}$ and $n$ in $\mathbb{N}$ one has

$$
w_{t_{1}, \ldots, t_{n}}\left(A_{1}, \ldots, A_{n} ; B_{1}, \ldots, B_{n}\right)=w_{t_{1}+t, \ldots, t_{n}+t}\left(A_{1}, \ldots, A_{n} ; B_{1}, \ldots, B_{n}\right),
$$

or, equivalently, if there exists a group $\left\{\alpha_{t}: t \in \mathbb{R}\right\}$ of $*$-automorphisms of $\mathscr{A}$ such that $j_{t}=\alpha_{t} j_{0}, \mu=\mu \circ \alpha_{t}$ for all $t$ in $\mathbb{R}$.

Given a stochastic process $\left(\mathscr{A},\left\{j_{t}: t \in \mathbb{R}\right\}, \mu\right)$, for any subset $I$ of $\mathbb{R}$, let $\mathscr{A}_{I}$ denote the $W^{*}$-subalgebra of $\mathscr{A}$ generated by $j_{t}(\mathscr{B}): t \in I$. Then the process is said to be Markov if, for each $t$ in $\mathbb{R}$, there exists a conditional expectation $E_{(-\infty, t]}$ of $\mathscr{A}$ onto $\mathscr{A}_{(-\infty, t]}$, which is compatible with $\mu$ in the sense that

$$
\mu=\left(\mu \uparrow \mathscr{A}_{(-\infty, t]}\right) \circ E_{(-\infty, t]},
$$

and satisfies

$$
E_{(-\infty, t]}\left(\mathscr{A}_{[t,+\infty)}\right)=\mathscr{A}_{\{t\}} \text {. }
$$

A stochastic dilation of a dynamical semigroup $\Phi_{t}$ on $\mathscr{B}$ is a stochastic process $\left(\mathscr{A},\left\{j_{t}: t \in \mathbb{R}\right\}, \mu\right)$ over $\mathscr{B}$ such that $j_{0}$ is invertible, and there exists a conditional expectation $E_{\{0\}}$ of $\mathscr{A}$ onto $\mathscr{A}_{\{0\}}$, compatible with $\mu$, satisfying

$$
\begin{aligned}
& j_{0}^{-1} E_{\{0\}}\left(j_{t_{1}}\left(A_{1}^{*}\right) \ldots j_{t_{n}}\left(A_{n}^{*}\right) j_{t_{n}}\left(B_{n}\right) \ldots j_{t_{1}}\left(B_{1}\right)\right) \\
& \quad=\Phi_{t_{1}}\left(A_{1}^{*} \Phi_{t_{2}-t_{1}}\left(A_{2}^{*} \ldots \Phi_{t_{n}-t_{n-1}}\left(A_{n}^{*} B_{n}\right) \ldots B_{2}\right) B_{1}\right),
\end{aligned}
$$

for all $A_{1}, \ldots, A_{n}, B_{1}, \ldots, B_{n}$ in $\mathscr{B}, 0 \leqq t_{1} \leqq t_{2} \leqq \ldots \leqq t_{n}$ in $\mathbb{R}$, and $n$ in $\mathbb{N}$. A stationary Markov dilation [12-14] of a dynamical semigroup $\Phi_{t}$ on $\mathscr{B}$ is a stationary Markov $W^{*}$-stochastic process $\left(\mathscr{A},\left\{j_{t}: t \in \mathbb{R}\right\}, \mu\right)$ over $\mathscr{B}$ such that there exists a normal conditional expectation $E_{\{0\}}$ of $\mathscr{A}$ onto $\mathscr{A}_{\{0\}}$, compatible with $\mu$, satisfying

$$
\Phi_{t}=j_{0}^{-1} E_{\{0\}} j_{t}\left(=j_{0}^{-1} E_{(-\infty, 0 j} j_{t}\right) \text { for all } t \text { in } \mathbb{R}^{+} \text {. }
$$

A stationary Markov dilation is both a stochastic dilation [12,9] and a unitary dilation in the sense of the Introduction.

\section{Stochastic Dilations}

Let $\Phi_{t}=\exp L t$ be a dynamical semigroup of finite Lindblad type on a $W^{*}$-algebra $\mathscr{B}$, describing the irreversible time evolution of a quantum system. We have

$$
L(B)=i[H, B]+\sum_{j=1}^{N} V_{j}^{*} B V_{j}-\frac{1}{2}\left[V_{j}^{*} V_{j}, B\right]_{+}
$$


for all $B$ in $\mathscr{B}$, where $H=H^{*} \in \mathscr{B}, V_{j} \in \mathscr{B}, j=1, \ldots, N$. We construct a stochastic dilation of $\Phi_{t}$ by coupling the system to a suitable reservoir and taking the singular coupling limit, as in $[2,10]$.

Let $\mathscr{F}$ be the antisymmetric Fock space over the direct sum of $N$ copies of $L^{2}(\mathbb{R})$. Denote by $\Omega$ the vacuum vector, and by $a_{j}(f), a_{j}(f)^{*}$ the annihilation and creation operators for a fermion with wave-function $f$ in the $j^{\text {th }}$ copy of $L^{2}(\mathbb{R})$. Let $\left\{\alpha_{t}^{0}: t \in \mathbb{R}\right\}$ be the weakly * continuous group of normal *automorphisms of $\mathscr{A} \equiv \mathscr{B} \otimes \mathscr{B}(\mathscr{F})$, determined by

$$
\alpha_{t}^{0}\left(B \otimes a_{j}(f)\right)=\mathrm{e}^{i H t} B \mathrm{e}^{-i H t} \otimes a_{j}\left(f_{t}\right)
$$

for all $B$ in $\mathscr{B}, f$ in $L^{2}(\mathbb{R}), j$ in $\{1, \ldots, N\}$ and $t$ in $\mathbb{R}$, where $H=H^{*}$ is the same as in (3.1), and where $f_{t}(s)=f(s-t)$ for all $f$ in $L^{2}(\mathbb{R})$ and all $s, t$ in $\mathbb{R}$.

Let $\left\{f^{\varepsilon}: \varepsilon>0\right\}$ be a family of real-valued test functions in $L^{2}(\mathbb{R})$, such that

$$
h^{\varepsilon}(t-s)=\left(f_{s}^{\varepsilon}, f_{t}^{\varepsilon}\right) ; \quad s, t \in \mathbb{R}, \quad \varepsilon>0,
$$

defines a positive symmetric function $h^{\varepsilon}$ in $L^{1}(\mathbb{R})$, with $\left\|h^{\varepsilon}\right\|_{1}$ independent of $\varepsilon$, and such that

$$
\lim _{\varepsilon \rightarrow 0} \int_{\mathbb{R}} g(s) h^{\varepsilon}(t-s) \mathrm{d} s=g(t)
$$

for all continuous and bounded functions $g$ on $\mathbb{R}$ and all $t$ in $\mathbb{R}$ (for example, let $\hat{f}^{\varepsilon}(\omega)=(2 \pi)^{-1 / 2} \exp \left[-\varepsilon^{2} \omega^{2} / 8\right]$, leading to $\left.h^{\varepsilon}(t)=\varepsilon^{-1} \pi^{-1 / 2} \exp \left[-t^{2} / \varepsilon^{2}\right]\right)$.

Let $V^{\varepsilon}$ be the self-adjoint element of $\tilde{\mathscr{A}}$ defined by

$$
V^{\varepsilon}=\sum_{j=1}^{N} V_{j}^{*} \otimes a_{j}\left(f^{\varepsilon}\right)+V_{j} \otimes a_{j}\left(f^{\varepsilon}\right)^{*} .
$$

The integral equation

$$
\alpha_{t}^{\varepsilon}(A)=\alpha_{t}^{0}(A)+i \int_{0}^{t} \alpha_{t-s}^{0}\left(\left[V^{\varepsilon}, \alpha_{s}^{\varepsilon}(A)\right]\right) \mathrm{d} s,
$$

$A \in \tilde{\mathscr{A}}$, may be solved by iteration (Dyson series), and defines a weakly* continuous group $\left\{\alpha_{t}^{\varepsilon}: t \in \mathbb{R}\right\}$ of normal ${ }^{*}$-automorphisms of $\tilde{\mathscr{A}}$. For each $t$ in $\mathbb{R}$, define a faithful normal ${ }^{*}$-representation $j_{t}^{\varepsilon}$ of $\mathscr{B}$ into $\tilde{\mathscr{A}}$ by

$$
j_{t}^{\varepsilon}(B)=\alpha_{t}^{\varepsilon}(B \otimes \mathbb{1})=U_{t}^{\varepsilon}(B \otimes \mathbb{1}) U_{t}^{\varepsilon *}
$$

for all $B$ in $\mathscr{B}$ and $t$ in $\mathbb{R}$, where $U_{t}^{\varepsilon} \in \tilde{\mathscr{A}}$ is the solution of the differential equation

$$
\frac{\mathrm{d}}{\mathrm{d} t} U_{t}^{\varepsilon}=i U_{t}^{\varepsilon}\left[H+\sum_{j=1}^{N} V_{j}^{*} \otimes a_{j}\left(f_{t}^{\varepsilon}\right)+V_{j} \otimes a_{j}\left(f_{t}^{\varepsilon}\right)^{*}\right], \quad U_{0}^{\varepsilon}=\mathbb{1} .
$$

Finally, denote by $F_{0}$ the map of $\tilde{\mathscr{A}}$ onto $\mathscr{B}$ defined by

$$
F_{0}(B \otimes A)=(\Omega, A \Omega) B, \quad B \in \mathscr{B}, \quad A \in \mathscr{B}(\mathscr{F}) .
$$

Theorem 3.1 (Dümcke $[10,11]$ ). For all $B_{1}, \ldots, B_{n}$ in $\mathscr{B}, t_{1}, \ldots, t_{n}$ in $\mathbb{R}$ and $n$ in $\mathbb{N}$, the expressions

$$
F_{0}\left(j_{t_{1}}^{\varepsilon}\left(B_{1}\right) \ldots j_{t_{n}}^{\varepsilon}\left(B_{n}\right)\right)
$$


converge in norm in the limit as $\varepsilon \rightarrow 0$, uniformly for $B_{1}, \ldots, B_{n}$ in a compact ball, $t_{1}, \ldots, t_{n}$ in a compact interval, and $n$ in a finite set. The limit may be computed by means of a term-by-term evaluation of a uniformly convergent Dyson series. Moreover, for "pyramidal" time ordering, $0 \leqq t_{1} \leqq \ldots \leqq t_{n}$, we have

$$
\begin{aligned}
& \lim _{\varepsilon \rightarrow 0} F_{0}\left(j_{t_{1}}^{\varepsilon}\left(A_{1}^{*}\right) \ldots j_{t_{n}}^{\varepsilon}\left(A_{n}^{*}\right) j_{t_{n}}^{\varepsilon}\left(B_{n}\right) \ldots j_{t_{1}}^{\varepsilon}\left(B_{1}\right)\right) \\
& \quad=\Phi_{t_{1}}\left(A_{1}^{*} \Phi_{t_{2}-t_{1}}\left(A_{2}^{*} \ldots \Phi_{t_{n}-t_{n-1}}\left(A_{n}^{*} B_{n}\right) \ldots B_{2}\right) B_{1}\right)
\end{aligned}
$$

for all $A_{1}, \ldots, A_{n}, B_{1}, \ldots, B_{n}$ in $\mathscr{B}$.

Proof. See [10] for the case of pyramidal time ordering and the Appendix for the general case.

Let $\mathscr{A}^{\varepsilon}$ be the $W^{*}$-subalgebra of $\tilde{\mathscr{A}}$ generated by $j_{t}^{\varepsilon}(\mathscr{B}): t \in \mathbb{R}$. Let $\varrho$ be a normal state on $\mathscr{B}$ such that the GNS representation $\pi_{\varrho}$ is faithful, and let $\mu^{\varepsilon}$ be the restriction of $\varrho \circ F_{0}$ to $\mathscr{A}^{\varepsilon}$. Then we have

Theorem 3.2. The correlation kernels $w_{t_{1}, \ldots, t_{n}}^{\varepsilon}$ of the quantum stochastic process $\left(\mathscr{A}^{\varepsilon},\left\{j_{t}^{\varepsilon}: t \in \mathbb{R}\right\}, \mu^{\varepsilon}\right)$ converge in the limit as $\varepsilon \rightarrow 0$ to the correlation kernels $w_{t_{1}}, \ldots, t_{n}$ of a quantum stochastic process $\left(\mathscr{A},\left\{j_{t}: t \in \mathbb{R}\right\}, \mu\right)$, which is a stochastic dilation of $\Phi_{t}=\exp L t$.

Proof. For all $A_{1}, \ldots, A_{n}, B_{1}, \ldots, B_{n}$ in $\mathscr{B}, t_{1}, \ldots, t_{n}$ in $\mathbb{R}$ and $n$ in $\mathbb{N}$, the expression

$$
w_{t_{1}, \ldots, t_{n}}^{\varepsilon}\left(A_{1}, \ldots, A_{n} ; B_{1}, \ldots, B_{n}\right)=\varrho\left(F_{0}\left[j_{t_{1}}^{\varepsilon}\left(A_{1}^{*}\right) \ldots j_{t_{n}}^{\varepsilon}\left(A_{n}^{*}\right) j_{t_{n}}^{\varepsilon}\left(B_{n}\right) \ldots j_{t_{1}}^{\varepsilon}\left(B_{1}\right)\right]\right)
$$

converges in the limit as $\varepsilon \rightarrow 0$, by Theorem 3.1; denote its limit by $w_{t_{1}, \ldots, t_{n}}\left(A_{1}, \ldots, A_{n} ; B_{1}, \ldots, B_{n}\right)$. This defines a family $\left\{w_{t_{1}, \ldots, t_{n}}: t_{1}, \ldots, t_{n} \in \mathbb{R}, n \in \mathbb{N}\right\}$ of functions

$$
w_{t_{1}, \ldots, t_{n}}:(\underbrace{\mathscr{B} \times \ldots \times \mathscr{B}}_{n \text { times }}) \times(\underbrace{\mathscr{B} \times \ldots \times \mathscr{B}}_{n \text { times }}) \rightarrow \mathbb{C},
$$

which inherit from $\left\{w_{t_{1}}^{\varepsilon}, \ldots, t_{n}: t_{1}, \ldots, t_{n} \in \mathbb{R}, n \in \mathbb{N}\right\}$ the following properties:

$C K 1$ (projectivity): If $A_{k}=B_{k}=\mathbb{1}, k=1, \ldots, n$, then

$w_{t_{1}, \ldots, t_{n}}\left(A_{1}, \ldots, A_{n} ; B_{1}, \ldots, B_{n}\right)=w_{t_{1}, \ldots, \hat{t}_{k}, \ldots, t_{n}}\left(A_{1}, \ldots, \hat{A}_{k}, \ldots, A_{n} ; B_{1}, \ldots, \hat{B}_{k}, \ldots, B_{n}\right)$,

where the marking ^above a symbol indicates that it must be omitted;

$C K 2$ (positivity): $w_{t_{1}, \ldots, t_{n}}$ is a positive definite kernel [4] on $(\mathscr{B} \times \ldots \times \mathscr{B})$ $\times(\mathscr{B} \times \ldots \times \mathscr{B})$

$C K 3$ (normalization): $w_{t}(\mathbb{1}, \mathbb{1})=1$;

CK4 (sesquilinearity): $w_{t_{1}, \ldots, t_{n}}$ is linear in the last $n$ arguments and conjugatelinear in the first $n$ arguments;

CK5 (*-condition): the map $A_{n}, B_{n} \mapsto w_{t_{1}}, \ldots, t_{n}\left(A_{1}, \ldots, A_{n} ; B_{1}, \ldots, B_{n}\right)$ of $\mathscr{B} \times \mathscr{B}$ into $\mathbb{C}$ factors through the map $A_{n}, B_{n} \mapsto A_{n}^{*} B_{n}$ of $\mathscr{B} \times \mathscr{B}$ into $\mathscr{B}$;

CK6 (multiplicativity): if $t_{k}=t_{k-1}, k=2, \ldots, n$, we have

$$
\begin{aligned}
& w_{t_{1}, \ldots, t_{n}}\left(A_{1}, \ldots, A_{n} ; B_{1}, \ldots, B_{n}\right) \\
& \quad=w_{t_{1}, \ldots, \hat{t}_{k}, \ldots, t_{n}}\left(A_{1}, \ldots, A_{k} A_{k-1}, \ldots, A_{n} ; B_{1}, \ldots, B_{k} B_{k-1}, \ldots, B_{n}\right) .
\end{aligned}
$$


These are the hypotheses of the reconstruction theorem of [8], hence there exists a stochastic process $\left(\mathscr{A},\left\{j_{t}: t \in \mathbb{R}\right\}, \mu\right)$, unique up to equivalence, such that (2.4) holds.

We regard $\mathscr{A}$ as a von Neumann algebra of operators on a Hilbert space $\mathscr{H}$, with a cyclic vector $\xi$ such that $(\xi, A \xi)=\mu(A)$ for all $A$ in $\mathscr{A}$. Denote by $P_{0}$ the orthogonal projection of $\mathscr{H}$ onto $\mathscr{H}_{0} \equiv \bar{j}_{0}(\mathscr{B}) \xi$, and let $\pi_{0}(B): B \in \mathscr{B}$ denote the restriction of $j_{0}(B)$ to $\mathscr{H}_{0}$. Since $\mu\left(j_{0}(B)\right)=\varrho(B)$ for all $B$ in $\mathscr{B}$, the triple $\left(\mathscr{H}_{0}, \pi_{0}, \xi\right)$ is a realization of the GNS representation associated with the normal state $\varrho$; then $\pi_{0}$ is normal, and faithful by assumption. Hence also $j_{0}$ is invertible.

Now we prove that $P_{0} \mathscr{A} P_{0} \subseteq \pi_{0}(\mathscr{B})$. Since $P_{0}(\cdot) P_{0}$ is a normal linear map and $\pi_{0}(\mathscr{B})$ is a von Neumann algebra, it suffices to prove that $P_{0} A P_{0} \in \pi_{0}(\mathscr{B})$ for $A$ of the form

$$
A=j_{t_{1}}\left(A_{1}^{*}\right) \ldots j_{t_{n}}\left(A_{n}^{*}\right) j_{t_{n}}\left(B_{n}\right) \ldots j_{t_{1}}\left(B_{1}\right): A_{1}, \ldots, A_{n}, B_{1}, \ldots, B_{n} \in \mathscr{B}, t_{1}, \ldots, t_{n} \in \mathbb{R}, n \in \mathbb{N},
$$

since the linear span of such $A$ 's is weakly* dense in $\mathscr{A}$. For all $\varphi=j_{0}\left(A_{0}\right) \xi$, $\psi=j_{0}\left(B_{0}\right) \xi: A_{0}, B_{0} \in \mathscr{B}$, we have

$$
\begin{aligned}
\left(\varphi, P_{0} A P_{0} \psi\right) & =\lim _{\varepsilon \rightarrow 0} w_{0, t_{1}, \ldots, t_{n}}^{\varepsilon}\left(A_{0}, A_{1}, \ldots, A_{n} ; B_{0}, B_{1}, \ldots, B_{n}\right) \\
& =\varrho\left(A_{0}^{*} \lim _{\varepsilon \rightarrow 0} F_{0}\left[j_{t_{1}}^{\varepsilon}\left(A_{1}^{*}\right) \ldots j_{t_{n}}^{\varepsilon}\left(A_{n}^{*}\right) j_{t_{n}}^{\varepsilon}\left(B_{n}\right) \ldots j_{t_{1}}^{\varepsilon}\left(B_{1}\right)\right] B_{0}\right) \\
& =\left(\varphi, \pi_{0}\left(\lim _{\varepsilon \rightarrow 0} F_{0}\left[j_{t_{1}}^{\varepsilon}\left(A_{1}^{*}\right) \ldots j_{t_{n}}^{\varepsilon}\left(A_{n}^{*}\right) j_{t_{n}}^{\varepsilon}\left(B_{n}\right) \ldots j_{t_{1}}^{\varepsilon}\left(B_{1}\right)\right]\right) \psi\right),
\end{aligned}
$$

where we have used $j_{0}^{\varepsilon}(B)=B \otimes \mathbb{1}$ for all $\varepsilon$. Since (3.12) holds for $\varphi, \psi$ in a dense set in $\mathscr{H}_{0}$, this proves $P_{0} \mathscr{A} P_{0} \cong \pi_{0}(\mathscr{B})$.

Then we define a map $E_{\{0\}}$ on $\mathscr{A}$ by

$$
E_{\{0\}}(A)=j_{0} \pi_{0}^{-1}\left(P_{0} A P_{0}\right): \quad A \in \mathscr{A} .
$$

$E_{\{0\}}$ is a norm one projection of $\mathscr{A}$ onto $\mathscr{A}_{\{0\}}$, hence a conditional expectation, by Tomiyama's theorem (see e.g. Theorem 5.3 of [4]). It follows from (3.12), in the particular case $\varphi=\psi=\xi$, that

$$
\mu(A)=\varrho \circ j_{0}^{-1} E_{\{0\}}(A) \text { for all } A \text { in } \mathscr{A},
$$

so that $E_{\{0\}}$ is compatible with $\mu$. For all $A_{1}, \ldots, A_{n}, B_{1}, \ldots, B_{n}$ in $\mathscr{B}, 0 \leqq t_{1} \leqq \ldots \leqq t_{n}$ in $\mathbb{R}$ and $n$ in $\mathbb{N}$, we have, from (3.12) and (3.11),

$$
\begin{aligned}
& E_{\{0\}}\left(j_{t_{1}}\left(A_{1}^{*}\right) \ldots j_{t_{n}}\left(A_{n}^{*}\right) j_{t_{n}}\left(B_{n}\right) \ldots j_{t_{1}}\left(B_{1}\right)\right) \\
& \quad=j_{0}\left(\Phi_{t_{1}}\left(A_{1}^{*} \Phi_{t_{2}-t_{1}}\left(A_{2}^{*} \ldots \Phi_{t_{n}-t_{n-1}}\left(A_{n}^{*} B_{n}\right) \ldots B_{2}\right) B_{1}\right) .\right.
\end{aligned}
$$

This concludes the proof that $\left(\mathscr{A},\left\{j_{t}: t \in \mathbb{R}\right\}, \mu\right)$ is a stochastic dilation of $\Phi_{t}$.

Theorem 3.3. The process $\left(\mathscr{A},\left\{j_{t}: t \in \mathbb{R}\right\}, \mu\right)$ satisfies the following "Markov property at $t=0$ ": for all $A_{-}$in $\mathscr{A}_{(-\infty, 0]}$ and $A_{+}$in $\mathscr{A}_{[0,+\infty)}$, one has

$$
\mu\left(A_{-} A_{+}\right)=\mu\left(E_{\{0\}}\left(A_{-}\right) A_{+}\right)=\mu\left(A_{-} E_{\{0\}}\left(A_{+}\right)\right)=\mu\left(E_{\{0\}}\left(A_{-}\right) E_{\{0\}}\left(A_{+}\right)\right),
$$

where $E_{\{0\}}$ is the conditional expectation of $\mathscr{A}$ onto $\mathscr{A}_{\{0\}}$ defined by (3.13). 
Proof. It suffices to show that, for all $s_{1}, \ldots, s_{m}$ in $\mathbb{R}^{-}, t_{1}, \ldots, t_{n}$ in $\mathbb{R}^{+}, A_{1}, \ldots, A_{m}$, $B_{1}, \ldots, B_{n}$ in $\mathscr{B}$, and $m, n$ in $\mathbb{N}$, one has

$$
\begin{aligned}
& \lim _{\varepsilon \rightarrow 0} F_{0}\left[j_{s_{1}}^{\varepsilon}\left(A_{1}\right) \ldots j_{s_{m}}^{\varepsilon}\left(A_{m}\right) j_{t_{1}}^{\varepsilon}\left(B_{1}\right) \ldots j_{t_{n}}^{\varepsilon}\left(B_{n}\right)\right] \\
& \quad=\left(\lim _{\varepsilon \rightarrow 0} F_{0}\left[j_{s_{1}}^{\varepsilon}\left(A_{1}\right) \ldots j_{s_{m}}^{\varepsilon}\left(A_{m}\right)\right]\right)\left(\lim _{\varepsilon \rightarrow 0} F_{0}\left[j_{t_{1}}^{\varepsilon}\left(B_{1}\right) \ldots j_{t_{n}}^{\varepsilon}\left(B_{n}\right)\right]\right) .
\end{aligned}
$$

By Theorem 3.1, we may evaluate the limit on the Dyson expansion (see Appendix). Taking into account Eq. (A.10), it suffices to prove that

$$
\lim _{\varepsilon \rightarrow 0} \int_{u=s}^{0} \int_{v=0}^{t}\left(f_{u}^{\varepsilon}, f_{v}^{\varepsilon}\right) \mathrm{d} u \mathrm{~d} v=0 \text { for all } s<0<t .
$$

Indeed, let $v-u=|v|+|u|=x, v+u=y$ : then $|y| \leqq x$ and the double integral in (3.15) becomes

$$
\frac{1}{2} \int_{x=0}^{|s|+t} \int_{y=-x}^{x} h^{\varepsilon}(x) \mathrm{d} y \mathrm{~d} x=\int_{0}^{|s|+t} h^{\varepsilon}(x) x \mathrm{~d} x=\frac{1}{2} \int_{-|s|-t}^{|s|+t} h^{\varepsilon}(x)|x| \mathrm{d} x
$$

by the assumed symmetry of $h^{\varepsilon}$, this vanishes in the limit as $\varepsilon \rightarrow 0$, by (3.4), thus proving (3.15).

Remarks. (i) The construction works exactly in the same way also if the antisymmetric Fock space is replaced by the symmetric Fock space (boson reservoir); this leads to an inequivalent stochastic dilation of $\Phi_{t}$.

(ii) The boson construction should give the same process as the one obtained by Hudson and Parthasarathy $[17,11]$ by solving a noncommutative stochastic differential equation. It seems conceivable that our fermion construction could also be obtained with the use of the fermion stochastic integral of Streater et al. $[26,11]$.

(iii) An alternative approximation method ("multiplicative Itô integral") has been used by von Waldenfels $[18,11]$ in the construction of a quantum stochastic process modelling atomic radiation. When specialized to that model, our "Stratonovich-type" approximation method yields the same result as his "Itôtype" method, by the assumed symmetry of the function $h^{\varepsilon}(t)$.

(iv) To our knowledge, the first results of this kind were obtained by Davies in [27]; there, however, only the "outgoing states" of the "system plus reservoir" were constructed.

\section{Stationarity, Detailed Balance, and Markov Property}

In the present section we assume that the dynamical semigroup $\Phi_{t}$ has a faithful normal stationary state, and investigate necessary and sufficient conditions for the stationarity of its stochastic dilation, as constructed in the previous section.

Theorem 4.1. Let $\Phi_{t}=\exp L t$ be a dynamical semigroup of finite Lindblad type on $\mathscr{B}$, with a faithful normal stationary state $\varrho$. If the stochastic dilation $\left(\mathscr{A},\left\{j_{t}: t \in \mathbb{R}\right\}\right.$, $\left.\mu=\varrho \circ j_{0}^{-1} \circ E_{\{0\}}\right)$ is a stationary process, then $\Phi_{t}$ satisfies the detailed balance condition with respect to $\varrho$. 
Proof. We have $\Phi_{t}=j_{0}^{-1} \circ E_{\{0\}} \circ j_{t}, t \in \mathbb{R}^{+}$. Let also $\Phi_{t}^{+}$be the map on $\mathscr{B}$ defined by

$$
\Phi_{t}^{+}=j_{0}^{-1} \circ E_{\{0\}} \circ j_{-t}, \quad t \in \mathbb{R}^{+} \text {. }
$$

By the assumed stationarity of the process, we have

$$
\begin{aligned}
\varrho\left(A \Phi_{t}(B)\right) & =\mu\left(j_{0}(A) j_{t}(B)\right)=\mu\left(j_{0}(A) \alpha_{t}\left(j_{0}(B)\right)\right) \\
& =\mu\left(\alpha_{-t}\left(j_{0}(A)\right) j_{0}(B)\right)=\mu\left(j_{-t}(A) j_{0}(B)\right)=\varrho\left(\Phi_{t}^{+}(A) B\right)
\end{aligned}
$$

for all $A, B$ in $\mathscr{B}$. The same construction as in Theorem 3.1 proves that $\Phi_{t}^{+}=\exp L^{+} t$, where

$$
L^{+}(B)=-i[H, B]+\sum_{j=1}^{N} V_{j}^{*} B V_{j}-\frac{1}{2}\left[V_{j}^{*} V_{j}, B\right]_{+},
$$

for all $B$ in $\mathscr{B}$ (cf. [24, Sect. 8]). Then $L$ and $L^{+}$satisfy (2.2) and (2.3), and detailed balance holds.

Theorem 4.1 has a partial converse. Let $\varrho$ be a faithful normal state on a $W^{*}$ algebra $\mathscr{B}$, and denote by $\left\{\sigma_{t}: t \in \mathbb{R}\right\}$ the associated modular automorphism group. If $\mathscr{B}$ is the algebra of all $n \times n$ complex matrices for some integer $n$, then any dynamical semigroup on $\mathscr{B}$ is of finite Lindblad type, and it satisfies the detailed balance condition if and only if $[20,21]$ its infinitesimal generator $L$ may be expressed as

$$
\begin{aligned}
L(B)= & i[H, B]+\sum_{k=1}^{N}\left(V_{k}^{*} B V_{k}-\frac{1}{2}\left[V_{k}^{*} V_{k}, B\right]_{+}\right) \\
& +\mathrm{e}^{-\beta \omega_{k}}\left(V_{k} B V_{k}^{*}-\frac{1}{2}\left[V_{k} V_{k}^{*}, B\right]_{+}\right)
\end{aligned}
$$

for all $B$ in $\mathscr{B}$, where $H=H^{*} \in \mathscr{B}, V_{k} \in \mathscr{B}, k=1, \ldots, N$, and where

$$
\begin{gathered}
\sigma_{t}(H)=H \quad \text { for all } t \text { in } \mathbb{R}, \\
\sigma_{t}\left(V_{k}\right)=\mathrm{e}^{i \beta \omega_{k} t} V_{k}, \quad \omega_{k} \in \mathbb{R}, \text { for all } t \text { in } \mathbb{R}, \quad k=1, \ldots, N .
\end{gathered}
$$

For an arbitrary $W^{*}$-algebra $\mathscr{B}$, we assume (4.4)-(4.6) as the form of $L$. Then we have:

Theorem 4.2. Let $\Phi_{t}=\exp L t$ be a dynamical semigroup on $\mathscr{B}$, with $L$ of the form (4.4), satisfying conditions (4.5) and (4.6). Then

(i) $\Phi_{t}$ satisfies the detailed balance condition with respect to $\varrho$;

(ii) the stochastic dilation $\left(\mathscr{A},\left\{j_{t}: t \in \mathbb{R}\right\}, \mu=\varrho \circ j_{0}^{-1} \circ E_{\{0\}}\right)$ is a stationary process;

(iii) $\mu$ is a faithful state on $\mathscr{A}$.

Proof. (i) Using the KMS condition in the form $\varrho(A B)=\varrho\left(\sigma_{i}(B) A\right)$ for analytic elements $B$ of $\mathscr{B}, A$ in $\mathscr{B}$, we find that

$$
\varrho(A[i H, B])=-\varrho([i H, A] B),
$$

[by (4.5)];

$$
\begin{aligned}
& \varrho\left(A\left[V_{k}^{*} V_{k}, B\right]_{+}\right)=\varrho\left(\left[V_{k}^{*} V_{k}, A\right]_{+} B\right), \\
& \varrho\left(A\left[V_{k} V_{k}^{*}, B\right]_{+}\right)=\varrho\left(\left[V_{k} V_{k}^{*}, A\right]_{+} B\right),
\end{aligned}
$$


and

$$
\varrho\left(A V_{k}^{*} B V_{k}\right)=\mathrm{e}^{-\beta \omega_{k}} \varrho\left(V_{k} A V_{k}^{*} B\right),
$$

[by (4.6)];

for all $A, B$ in $\mathscr{B}$. Then (2.2) holds, with $L^{+}=L-2 i[H, \cdot]$.

(ii) It suffices to prove that the approximating processes $\left(\mathscr{A}^{\varepsilon},\left\{j_{t}^{\varepsilon}: t \in \mathbb{R}\right\}, \mu^{\varepsilon}\right)$ are stationary for all $\varepsilon$. We need the antisymmetric Fock space over the direct sum of $2 N$ copies of $L^{2}(\mathbb{R})$, which we label by indices $k= \pm 1, \ldots, \pm N$. From (3.5), we have

$$
\begin{aligned}
V^{\varepsilon}= & \sum_{k=1}^{N} V_{k}^{*} \otimes\left[a_{k}\left(f^{\varepsilon}\right)+\mathrm{e}^{-\beta \omega_{k} / 2} a_{-k}\left(f^{\varepsilon}\right)^{*}\right] \\
& +V_{k} \otimes\left[a_{k}\left(f^{\varepsilon}\right)^{*}+\mathrm{e}^{-\beta \omega_{k} / 2} a_{-k}\left(f^{\varepsilon}\right)\right] \\
= & \sum_{k=1}^{N}\left(1+\mathrm{e}^{-\beta \omega_{k}}\right)^{1 / 2}\left[V_{k}^{*} \otimes b_{k}\left(f^{\varepsilon}\right)+V_{k} \otimes b_{k}\left(f^{\varepsilon}\right)^{*}\right],
\end{aligned}
$$

where we have defined the Bogoliubov transformation

$$
b_{k}(f)=\left(1+\mathrm{e}^{-\beta \omega_{k}}\right)^{-1 / 2}\left[a_{k}(f)+\mathrm{e}^{-\beta \omega_{k} / 2} a_{-k}(\bar{f})\right]
$$

for $k=1, \ldots, N, f$ in $L^{2}(\mathbb{R})$ (recall that $f^{\varepsilon}$ is real-valued). It is well known [28] that the vacuum vector $\Omega$ is both cyclic and separating for the $W^{*}$-algebra $\mathscr{R}$ defined by

$$
\mathscr{R}=\left\{b_{k}(f), b_{k}(f)^{*}: k=1, \ldots, N, f \in L^{2}(\mathbb{R})\right\}^{\prime \prime},
$$

and that the associated modular automorphism group of $\mathscr{R}$ satisfies [29]

$$
\sigma_{t}\left(b_{k}(f)\right)=\mathrm{e}^{i \beta \omega_{k} t} b_{k}(f), \quad k=1, \ldots, N, \quad f \in L^{2}(\mathbb{R}) .
$$

Note that $\mathscr{B} \otimes \mathscr{R}$ is globally invariant under $\left\{\alpha_{t}^{0}: t \in \mathbb{R}\right\}$, and that $V^{\varepsilon}$ is in $\mathscr{B} \otimes \mathscr{R}$, so that $\left\{\alpha_{t}^{\varepsilon}: t \in \mathbb{R}\right\}$ maps $\mathscr{B} \otimes \mathscr{R}$ into itself, and $\mathscr{A}^{\varepsilon}$ is a $W^{*}$-subalgebra of $\mathscr{B} \otimes \mathscr{R}$. The state $\bar{\mu}=\varrho \circ F_{0} \uparrow \mathscr{B} \otimes \mathscr{R}$ is a faithful normal state, and it is invariant under $\left\{\alpha_{t}^{0}\right.$ : $t \in \mathbb{R}\}$, by (4.5). The associated modular automorphism group $\left\{\bar{\sigma}_{t}: t \in \mathbb{R}\right\}$ of $\mathscr{B} \otimes \mathscr{R}$ satisfies

$$
\bar{\sigma}_{t}\left(V^{\varepsilon}\right)=V^{\varepsilon} \text { for all } t \text { in } \mathbb{R}
$$

by (4.6) and (4.10). Then we have, for all $A$ in $\mathscr{A}^{\varepsilon}$ and $t$ in $\mathbb{R}$,

$$
\begin{aligned}
\bar{\mu}\left(\alpha_{t}^{\varepsilon}(A)\right)-\bar{\mu}(A) & =\bar{\mu}\left(\alpha_{t}^{\varepsilon}(A)-\alpha_{t}^{0}(A)\right) \\
& =i \int_{0}^{t} \bar{\mu}\left(\left[V^{\varepsilon}, \alpha_{s}^{\varepsilon}(A)\right]\right) \mathrm{d} s=0,
\end{aligned}
$$

by (3.6) and (4.11). Then $\left(\mathscr{A}^{\varepsilon},\left\{j_{t}^{\varepsilon}=\alpha_{t}^{\varepsilon} j_{0}: t \in \mathbb{R}\right\}, \mu^{\varepsilon}\right)$ is stationary.

(iii) By (ii) above, $\bar{\sigma}_{s}$ commutes with $\alpha_{t}^{\varepsilon}$ for all $s, t$ in $\mathbb{R}$ [30], hence $\bar{\sigma}_{s} j_{t}^{\varepsilon}(B)=j_{t}^{\varepsilon} \sigma_{s}(B)$ for all $s, t$ in $\mathbb{R}$ and $B$ in $\mathscr{B}$. Taking the limit of multi-time correlations as $\varepsilon \rightarrow 0$, we obtain

$$
\begin{aligned}
\int_{\mathbb{R}} & \mu\left(j_{t_{1}}\left(A_{1}\right) \ldots j_{t_{m}}\left(A_{m}\right) j_{u_{1}}\left(\sigma_{s}\left(B_{1}\right)\right) \ldots j_{u_{n}}\left(\sigma_{s}\left(B_{n}\right)\right)\right) f(s) \mathrm{d} s \\
& =\int_{\mathbb{R}} \mu\left(j_{u_{1}}\left(\sigma_{s}\left(B_{1}\right)\right) \ldots j_{u_{n}}\left(\sigma_{s}\left(B_{n}\right)\right) j_{t_{1}}\left(A_{1}\right) \ldots j_{t_{m}}\left(A_{m}\right)\right) f(s-i) \mathrm{d} s
\end{aligned}
$$

for all $A_{1}, \ldots, A_{m}, B_{1}, \ldots, B_{n}$ in $\mathscr{B}, s, t_{1}, \ldots, t_{m}, u_{1}, \ldots, u_{n}$ in $\mathbb{R}, m, n$ in $\mathbb{N}$, and for all functions $f$ with Fourier transform in $C_{0}^{\infty}(\mathbb{R})$. It follows, as in [31], that the pair 
$(\mathscr{A}, \mu)$ is equipped with a modular automorphism group $\left\{\sigma_{s}: s \in \mathbb{R}\right\}$, satisfying

$$
\sigma_{s}\left[j_{u_{1}}\left(B_{1}\right) \ldots j_{u_{n}}\left(B_{n}\right)\right]=j_{u_{1}}\left(\sigma_{s}\left(B_{1}\right)\right) \ldots j_{u_{n}}\left(\sigma_{s}\left(B_{n}\right)\right)
$$

for all $B_{1}, \ldots, B_{n}$ in $\mathscr{B}, s, u_{1}, \ldots, u_{n}$ in $\mathbb{R}$, and $n$ in $\mathbb{N}$; in particular, $\mu$ is a faithful state on $\mathscr{A}$.

We may conclude that the following statements are "essentially equivalent" (up to technicalities):

(I) $\Phi_{t}$ satisfies detailed balance with respect to $\varrho$;

(II) the stochastic dilation $\left(\mathscr{A},\left\{j_{t}: t \in \mathbb{R}\right\}, \mu\right)$ as constructed from $\Phi_{t}$ and $\varrho$ as in Sect. 3 is a stationary process, and $\mu$ is a faithful state on $\mathscr{A}$.

By using the modular automorphism group of $\mathscr{A}$ associated with $\mu$, the stationarity of the process, and Takesaki's theorem on conditional expectations [25], we can extend the "Markov property at $t=0$ " of Theorem 3.3 to the full Markov property.

Theorem 4.3. Let $\left(\mathscr{A},\left\{j_{t}: t \in \mathbb{R}\right\}, \mu\right)$ be the stochastic dilation, as in Sect. 3, of a dynamical semigroup $\Phi_{t}$ on $\mathscr{B}$. If it is a stationary process and $\mu$ is a faithful state, then it is a stationary Markov dilation of $\Phi_{t}$.

Proof. It remains to prove that $j_{t}$ is a faithful normal map for all $t$ in $\mathbb{R}, E_{\{0\}}$ is a normal conditional expectation, and the Markov property (2.7) holds. We give the proofs separately:

(i) Since $j_{t}=\alpha_{t} j_{0}$ and $\alpha_{t}$ is an automorphism leaving the normal state $\mu$ invariant, it suffices to prove that $j_{0}$ is normal ( $j_{0}$ is faithful by Theorem 3.2). Since $\mu$ is faithful, the set of the positive linear functionals on $\mathscr{A}$ that are dominated by a scalar multiple of $\mu$ generates a dense subset of $\mathscr{A}_{*}$. Let $v$ be such a positive functional. Then $v \circ j_{0}$ is dominated by a scalar multiple of $\mu \circ j_{0}=\varrho$, hence it is a normal functional on $\mathscr{B}$. By taking linear combinations and norm limits, it follows that $j_{0}^{*}$ maps $\mathscr{A}_{*}$ into $\mathscr{B}_{*}$, so that $j_{0}$ is normal.

(ii) $E_{\{0\}}$ satisfies $\mu \circ E_{\{0\}}=\varrho \circ j_{0}^{-1} \circ E_{\{0\}}=\mu$; and since $E_{\{0\}}$ is completely positive and $\mu$ is a faithful normal state, it follows that $E_{\{0\}}$ is normal.

(iii) The modular automorphism group $\left\{\sigma_{s}: s \in \mathbb{R}\right\}$ of $\mathscr{A}$ associated with $\mu$ commutes with $\left\{\alpha_{t}: t \in \mathbb{R}\right\}[30]$, hence all the $W^{*}$-subalgebras $\mathscr{A}_{I}=\vee\left\{\alpha_{t} j_{0}(\mathscr{B})\right.$ : $t \in I\}$, are globally invariant under $\sigma_{s}$. By [25], there exist conditional expectations $E_{I}$ of $\mathscr{A}$ onto $\mathscr{A}_{I}$ which are compatible with $\mu$; they are uniquely defined, faithful and normal. In particular, $E_{(-\infty, 0]}$ exists. In order to prove that (2.7) holds for $t=0$, it suffices to show that, for each $A_{+}$in $\mathscr{A}_{[0,+\infty)}$, there exists $A_{0}$ in $\mathscr{A}_{\{0\}}$ such that

$$
\mu\left(A_{-} A_{+}\right)=\mu\left(A_{-} A_{0}\right) \text { for all } A_{-} \text {in } \mathscr{A}_{(-\infty, 0]} \text {. }
$$

By Theorem 3.3, (4.13) holds, with $A_{0}=E_{\{0\}}\left(A_{+}\right)$. Then $E_{(-\infty, 0]}\left(\mathscr{A}_{[0,+\infty)}\right)=\mathscr{A}_{\{0\}}$. By the stationarity of the process, we have $\alpha_{t} E_{(-\infty, 0]}=E_{(-\infty, t]} \alpha_{t}$, and (2.7) follows.

Remarks: (i) The connection between stationarity of the dilation and the detailed balance condition for the semigroup should be compared with the "derivation of detailed balance from microscopic reversibility." As remarked in [24, 32], such a derivation is reliable only when the stationary state of the semigroup is the restriction of a stationary (or, at least, approximately stationary) state for the global evolution of the larger system. 
(ii) The processes we have described are called "quantum diffusions" by Hudson and Parthasarathy [17], in that they can be constructed with the aid of a "quantum Wiener process." It is worth mentioning, in this regard, that a classical diffusion with a stationary state does indeed satisfy the detailed balance condition in the sense of [21], as remarked in [32].

\section{Appendix}

A published proof of Theorem 3.1 exists only for the case of pyramidal time ordering [10]. Though the convergence of all multi-time correlations is an explicitly stated result in Dümcke's paper in [11], we think it convenient to provide an explicit proof here.

We introduce some notation. For $m$ in $\mathbb{N}$ and $t$ in $\mathbb{R}$, let

$$
\Delta_{m}(0, t)= \begin{cases}\left\{\left(s_{1}, \ldots, s_{m}\right) \in \mathbb{R}^{m}: 0 \leqq s_{1} \leqq \ldots \leqq s_{m} \leqq t\right\} & \text { for } t \geqq 0, \\ \left\{\left(s_{1}, \ldots, s_{m}\right) \in \mathbb{R}^{m}: t \leqq s_{1} \leqq \ldots \leqq s_{m} \leqq 0\right\} & \text { for } t \leqq 0\end{cases}
$$

We shall also understand that

$$
\sum_{m=0}^{\infty} \int_{\Delta_{m}(0, t)} f_{m}\left(t ; s_{1}, \ldots, s_{m}\right) \mathrm{d} s_{1} \ldots \mathrm{d} s_{m}=f_{0}(t)+\sum_{m=1}^{\infty} \int_{\Delta_{m}(0, t)} f_{m}\left(t ; s_{1}, \ldots, s_{m}\right) \mathrm{d} s_{1} \ldots \mathrm{d} s_{m} .
$$

For $A$ in $\tilde{\mathscr{A}}$ and $t$ in $\mathbb{R}$, let $A(t)=\alpha_{t}^{0}(A)$. It is convenient to rewrite $V^{\varepsilon}$ as

$$
V^{\varepsilon}=\sum_{j=1}^{2 N} F_{j} \otimes \varphi_{j}\left(f^{\varepsilon}\right)
$$

where

$$
\left.\begin{array}{ll}
F_{j}=\frac{1}{2}\left(V_{j}^{*}+V_{j}\right), \varphi_{j}=a_{j}^{*}+a_{j} & \text { for } \quad j=1, \ldots, N, \\
F_{j}=\frac{i}{2}\left(V_{j-N}^{*}-V_{j-N}\right), \varphi_{j}=i\left(a_{j-N}^{*}-a_{j-N}\right) & \text { for } \quad j=N+1, \ldots, 2 N .
\end{array}\right\}
$$

We define also

$$
\left.\begin{array}{l}
F_{+j}(A)=F_{j} A, \varphi_{+j}(A)=\varphi_{j} A, \\
F_{-j}(A)=A F_{j}, \varphi_{-j}(A)=A \varphi_{j},
\end{array}\right\}
$$

for all $A$ in $\tilde{\mathscr{A}}, j=1, \ldots, 2 N$. For all $B_{1}, \ldots, B_{n}$ in $\mathscr{B}, t_{1}, \ldots, t_{n}$ in $\mathbb{R}$ and $n$ in $\mathbb{N}$, the iterative solution of (3.6) yields

$$
\begin{aligned}
F_{0}\left[j_{t_{1}}^{\varepsilon}\right. & \left.\left(B_{1}\right) \ldots j_{t_{n}}^{\varepsilon}\left(B_{n}\right)\right] \\
= & \sum_{r=0}^{\infty} \sum_{m_{1}+\ldots+m_{n}=r}\left[\prod_{k=1}^{n}\left(i \operatorname{sgn} t_{k}\right)^{m_{k}}\right]_{\Delta_{m_{1}}\left(0, t_{1}\right) \times \ldots \times \Delta_{m_{n}}\left(0, t_{n}\right) j_{1}, \ldots, j_{r}= \pm 1, \ldots, \pm 2 N} \sum_{n} \ldots \int \\
& \cdot\left[\prod_{k=1}^{r} \operatorname{sgn} j_{k}\right] \\
& \cdot\left[F_{j_{1}}\left(s_{1}\right) \ldots F_{j_{m_{2}}}\left(s_{m_{1}}\right) B_{1}\left(t_{1}\right)\right]\left[F_{j_{m_{1}+1}}\left(s_{m_{1}+1}\right) \ldots F_{j_{m_{1}+m_{2}}}\left(s_{m_{1}+m_{2}}\right) B_{2}\left(t_{2}\right)\right] \\
& \cdot \ldots\left[F_{j_{m_{1}}+\ldots+m_{n-1}+1}\left(s_{m_{1}+\ldots+m_{n-1}+1}\right) \ldots F_{j_{r}}\left(s_{r}\right) B_{n}\left(t_{n}\right)\right] \\
& \cdot\left(\Omega\left[\varphi_{j_{1}}\left(f_{s_{1}}^{\varepsilon}\right) \ldots \varphi_{j_{m_{1}}}\left(f_{s_{m_{2}}}^{\varepsilon}\right) \mathbb{1}\right]\right. \\
& \left.\cdot \ldots\left[\varphi_{j_{m_{1}}+\ldots+m_{n-1}+1}\left(f_{s_{m_{1}+}+\ldots+m_{n-1}+1}^{\varepsilon}\right) \ldots \varphi_{j_{r}}\left(f_{s_{r}}^{\varepsilon}\right) \mathbb{1}\right] \Omega\right) \mathrm{d} s_{1} \ldots \mathrm{d} s_{r} .
\end{aligned}
$$


To each ordered $r$-uple $\left(\alpha_{1}, \ldots, \alpha_{r}\right)=\left(\operatorname{sgn} j_{1}, \ldots, \operatorname{sgn} j_{r}\right)$ in $\{-1,+1\}^{r}$, there corresponds a permutation $\pi=\pi\left(\alpha_{1}, \ldots, \alpha_{r}\right)$ of $\{1, \ldots, r\}$ such that $(\Omega, \ldots, \Omega)$ in (A.6) becomes

$$
\left(\Omega, \varphi_{\left|j_{\pi(1) \mid}\right|}\left(f_{s_{\pi(1)}}^{\varepsilon}\right) \ldots \varphi_{\mid j_{\pi(r) \mid}}\left(f_{s_{\pi(r)}}^{\varepsilon}\right) \Omega\right) .
$$

This vanishes for $r$ odd, and for $r$ even, $r=2 l$, it becomes

$$
\sum_{p \in \mathscr{P}(l)}(\operatorname{sgn} p) \prod_{q=1}^{l}\left(\Omega, \varphi_{\left|j_{\pi_{\circ} p(2 q-1)}\right|}\left(f_{s_{\pi \circ p(2 q-1)}}^{\varepsilon}\right) \varphi_{\left|j_{\pi \circ p(2 q)}\right|}\left(f_{s_{\pi \circ p(2 q)}}^{\varepsilon}\right) \Omega\right),
$$

where $\mathscr{P}(l)$ is the set of those permutations of $\{1, \ldots, 2 l\}$ such that $p(2 q-1)<p(2 q)$, $p(2 q-1)<p(2 q+1)$ for all $q$, and $\operatorname{sgn} p$ is the parity of the permutation $p$. Taking into account (A.4), and defining

$$
\chi(j, k)=\left\{\begin{aligned}
1 & \text { for } j=k, \\
i & \text { for } j=k-N, \\
-i & \text { for } j=k+N, \\
0 & \text { otherwise }
\end{aligned}\right.
$$

we find that (A.7) becomes

$$
\sum_{p \in \mathscr{P}(l)}(\operatorname{sgn} p) \prod_{q=1}^{l} \chi\left(\left|j_{\pi \circ p(2 q-1)}\right|,\left|j_{\pi \circ p(2 q)}\right|\right) h^{\varepsilon}\left(S_{\pi \circ p(2 q)}-S_{\pi \circ p(2 q-1)}\right) .
$$

Combining (A.6) with (A.9), we find

$$
\begin{aligned}
F_{0}\left[j_{t_{1}}^{\varepsilon}\right. & \left.\left(B_{1}\right) \ldots j_{t_{n}}^{\varepsilon}\left(B_{n}\right)\right] \\
= & \sum_{l=0}^{\infty} \sum_{m_{1}+\ldots+m_{n}=2 l}\left[\prod_{k=1}^{n}\left(i \operatorname{sgn} t_{k}\right)^{m_{k}}\right]_{\Delta_{m_{1}}\left(0, t_{1}\right) \times \ldots \times \Delta_{m_{n}}\left(0, t_{n}\right) j_{1}, \ldots, j_{r}= \pm 1, \ldots, \pm 2 N} \sum \ldots \int \\
& \cdot\left[\prod_{k=1}^{2 l} \operatorname{sgn} j_{k}\right] \\
& \cdot\left[F_{j_{1}}\left(s_{1}\right) \ldots F_{j_{m_{1}}}\left(s_{m_{1}}\right) B_{1}\left(t_{1}\right)\right] \ldots\left[F_{j_{m_{1}}+\ldots+m_{n-1}+1}\left(s_{m_{1}+\ldots+m_{n-1}+1}\right)\right. \\
& \left.\cdot \ldots F_{j_{2 l}}\left(s_{2 l}\right) B_{n}\left(t_{n}\right)\right] \\
& \cdot \sum_{p \in \mathscr{P}(l)}(\operatorname{sgn} p) \prod_{q=1}^{l} \chi\left(\left|j_{\pi \circ p(2 q-1)}\right|, \mid j_{\pi \circ p(2 q)}\right) h^{\varepsilon}\left(s_{\pi \circ p(2 q)}-S_{\pi \circ p(2 q-1)}\right) \mathrm{d} s_{1} \ldots \mathrm{d} s_{2 l}, \quad \text { A }
\end{aligned}
$$

where $\pi=\pi\left(\operatorname{sgn} j_{1}, \ldots, \operatorname{sgn} j_{2 l}\right)$. The term-by-term limit as $\varepsilon \rightarrow 0$ of the expansion (A.10) is evaluated by recalling that $h^{\varepsilon}(t)$ is a symmetric approximation to $\delta(t)$. In order to prove that the expansion converges uniformly in $\varepsilon$, we perform some majorizations. Since $|\chi(j, k)| \leqq 1$ and $h^{\varepsilon}$ is a positive symmetric function, we have

$$
\left|\sum_{p \in \mathscr{P}(l)} \ldots h^{\varepsilon}(\ldots)\right| \leqq \frac{1}{2^{l} l !} \sum_{\sigma \in S(2 l)} \prod_{q=1}^{l} h^{\varepsilon}\left(S_{\pi \circ \sigma(2 q)}-S_{\pi \circ \sigma(2 q-1)}\right),
$$

where $S(2 l)$ is the set of all permutations of $\{1, \ldots, 2 l\}$. This is actually independent of $\pi$, and it may be rewritten as

$$
\frac{(2 l) !}{2^{l} l !} P_{S}\left[\prod_{q=1}^{l} h^{\varepsilon}\left(s_{2 q}-s_{2 q-1}\right)\right],
$$


where $P_{S}$ denotes the projection onto the totally symmetric functions of $s_{1}, \ldots, s_{2 l}$. Let also $\|F\|=\max \left\|F_{|j|}\right\|,\|B\|=\max \left\|B_{i}\right\|$. Then we find

$$
\begin{aligned}
\| \text { r.h.s. of }(\mathrm{A} .10) \| \leqq & \sum_{l=0}^{\infty} \sum_{m_{1}+\ldots+m_{n}=2 l} \int_{\Delta_{m_{1}}\left(0, t_{1}\right) \times \ldots \times \int_{m_{n}}\left(0, t_{n}\right)} \ldots \|^{2 l} \\
& \cdot(4 N)^{2 l}\|F\|^{2 l}\|B\|^{n} \frac{(2 l) !}{2^{l} l !} P_{S}\left[\prod_{q=1}^{l} h^{\varepsilon}\left(s_{2 q}-s_{2 q-1}\right)\right] \mathrm{d} s_{1} \ldots \mathrm{d} s_{2 l} .
\end{aligned}
$$

Since the integrand is totally symmetric in $s_{1}, \ldots, s_{2 l}$, it is also symmetric in all the groups of variables $\left(s_{1}, \ldots, s_{m_{1}}\right),\left(s_{m_{1}+1}, \ldots, s_{m_{1}+m_{2}}\right), \ldots\left(s_{m_{1}+\ldots+m_{n-1}+1}, \ldots, s_{2 l}\right)$. Hence we may replace

$$
\int_{\Delta_{m}(0, t)}[\ldots] \mathrm{d} s_{1} \ldots \mathrm{d} s_{m} \text { by } \frac{1}{m !} \int_{0}^{t} \ldots \int_{0}^{t}[\ldots] \mathrm{d} s_{1} \ldots \mathrm{d} s_{m},
$$

and obtain a further majorization by extending each integration from $t_{-}=\min \left(t_{k}, 0\right)$ to $t_{+}=\max \left(t_{k}, 0\right)$. Then we obtain

$$
\begin{aligned}
& \| \text { r.h.s. of }\left(\text { A.10) }\|\leqq\| B \|^{n} \sum_{l=0}^{\infty}\left(8 N^{2}\|F\|^{2}\right)^{l} \frac{1}{l !}\right. \\
& \cdot\left[\sum_{m_{1}+\ldots+m_{n}=2 l} \frac{(2 l) !}{m_{1} ! \ldots m_{n} !}\right] \\
& \cdot \int_{t_{-}}^{t_{+}} \ldots \int_{t_{-}}^{t_{+}} P_{S}\left[\prod_{q=1}^{l} h^{\varepsilon}\left(s_{2 q}-s_{2 q-1}\right)\right] \mathrm{d} s_{1} \ldots \mathrm{d} s_{2 l} \text {. }
\end{aligned}
$$

The integral is majorized by $\left(\left\|h^{\varepsilon}\right\|_{1}\left(t_{+}-t_{-}\right)\right)^{l}$, independent of $\varepsilon$, as in [1]. It remains to control the behaviour of

$$
C(n, r)=\sum_{m_{1}+\ldots+m_{n}=r} \frac{r !}{m_{1} ! \ldots m_{n} !} .
$$

Using $C(1, r)=1$ for all $r$, and the easily derived recursion formula

$$
C(n, r)=\sum_{s=0}^{r}\left(\begin{array}{l}
r \\
s
\end{array}\right) C(n-1, s)
$$

we find that $C(n, r) \leqq(r+1)^{n-1} 2^{(n-1) r}$, which is eventually dominated by $2^{n r}$ for fixed $n$ and $r \rightarrow \infty$. We conclude that the expansion (A.10) is dominated by

$$
(\text { constant })+\|B\|^{n} \sum_{l=0}^{\infty} \frac{1}{l !}\left[8 N^{2}\|F\|^{2} 4^{n}\|h\|_{1}\left(t_{+}-t_{-}\right)\right]^{l},
$$

which is a convergent series, independent of $\varepsilon$. Then we are allowed to take the limit term by term in (A.10). Note that (A.13) proves also the uniformness of the convergence as stated in Theorem 3.1.

Acknowledgements. The preparation of this work greatly benefited from the stimulating atmosphere of the Workshop on Quantum Probability and Applications to the Quantum Theory 
of Irreversible Processes, held in Rome, Villa Mondragone, September 6-11, 1982. There we had the opportunity of discussing with many of the authors of the papers referred to. We are also grateful to L. Accardi, R. L. Hudson, G. L. Sewell, and H. Spohn for stimulating discussions on different occasions.

\section{References}

1. Davies, E.B.: Markovian master equations. Commun. Math. Phys. 39, 91-110 (1974)

2. Gorini, V., Kossakowski, A.: $N$-level system in contact with a singular reservoir. J. Math. Phys. 17, 1298-1305 (1976);

Frigerio, A., Gorini, V.: N-level systems in contact with a singular reservoir. II. J. Math. Phys. 17, 2123-2127 (1976)

3. Davies, E.B.: Dilations of completely positive maps. J. London Math. Soc. (2) 17, 330-338 (1978)

4. Evans, D.E., Lewis, J.T.: Dilations of irreversible evolutions in algebraic quantum theory. Commun. Dublin Institute for Advanced studies Ser. A No. 24, 1977

5. Varilly, J.C.: Dilation of a non-quasifree dissipative evolution. Lett. Math. Phys. 5, 113-116 (1981)

6. Spohn, H.: Kinetic equations from Hamiltonian dynamics: Markovian limits. Rev. Mode. Phys. 52, 569-615 (1980)

7. Lindblad, G.: Non-Markovian quantum stochastic processes and their entropy. Commun. Math. Phys. 65, 281-294 (1979)

8. Accardi, L., Frigerio, A., Lewis, J.T.: Quantum stochastic processes. Publ. RIMS Kyoto Univ. 18, 97-133 (1982)

9) Lewis, J.T.: Quantum stochastic processes. I. Phys. Rep. 77, 339-349 (1981); Frigerio, A.: Quantum stochastic processes. II. Phys. Rep. 77, 351-358 (1981)

10. Dümcke, R.: Convergence of multi-time correlation functions in the weak and singular coupling limits. J. Math. Phys. 24, 311-315 (1983)

11. Accardi, L., Frigerio, A., Gorini, V. (eds.): Quantum Probability and applications to the quantum theory of irreversible processes - Proceedings, Rome, 1982. Lecture Notes in Mathematics, Vol. 1055. Berlin, Heidelberg, New York, Tokyo: Springer 1984

12. Kümmerer, B.: A dilation theory for completely positive operators on $W^{*}$-algebras. Thesis, Tübingen, 1982

13. Kümmerer, B.: Markov dilations of completely positive operators on $W^{*}$-algebras. Semesterbericht Funktionalanalysis Tübingen, Wintersemester 1981/1982, pp. 175-186;

Kümmerer, B.: A non-commutative example of a continuous Markov dilation. Semesterbericht Funktionalanalyse Tübingen, Wintersemester 1982/1983, pp. 61-91

14. Kümmerer, B., Schröder, W.: A Markov dilation of a non-quasifree Bloch evolution. Commun. Math. Phys. 90, 251-262 (1983)

15. Emch, G.G.: Generalized K-flows. Commun. Math. Phys. 49, 191-215 (1976)

16. Emch, G.G., Albeverio, S., Eckmann, J.P.: Quasi-free generalized K-flows. Rep. Math. Phys. 13, 73-85 (1978)

17. Hudson, R.L., Parthasarathy, K.R.: Quantum diffusions. In: Theory and applications of random fields. Lecture Notes in Control and Information Sciences, Vol. 49. Kallianpur (ed.). Berlin, Heidelberg, New York, Tokyo: Springer 1983, Noncommutative semimartingales and quantum diffusion processes adapted to Brownian motion, preprint; Quantum Itô's formula and stochastic evolutions (preprint)

18. Von Waldenfels, W.: Light emission and absorption as a quantum stochastic process. Preprint Heidelberg No. 176, 1982

19. Lindblad, G.: On the generators of quantum dynamical semigroups. Commun. Math. Phys. 48, 119-130 (1976)

20. Alicki, R.: On the detailed balance condition for non-Hamiltonian systems. Rep. Math. Phys. 10, 249-258 (1976) 
21. Kossakowski, A., Frigerio, A., Gorini, V., Verri, M.: Quantum detailed balance and KMS condition. Commun. Math. Phys. 57, 97-110 (1977)

22. Evans, D.E.: Completely positive quasi-free maps on the CAR algebra. Commun. Math. Phys. 70, 53-68 (1979)

23. Lindblad, G.: Gaussian quantum stochastic processes on the CCR algebra. Math. Phys. 20, 2081-2087 (1979)

24. Gorini, V., Frigerio, A., Verri, M., Kossakowski, A., Sudarshan, E.C.G.: Properties of quantum Markovian master equations. Rep. Math. Phys. 13, 149-173 (1978)

25. Takesaki, M.: Conditional expectations in von Neumann algebras. J. Funct. Anal. 9, 306321 (1972)

26. Barnett, C., Streater, R.F., Wilde, I.F.: The Itô-Clifford integral. J. Funct. Anal. 48, 172-212 (1982); Itô-Clifford integral II-stochastic differential equations. J. London Math. Soc. (2), 27, 333-384 (1983): The Itô-Clifford integral. III. The Markov property of solutions to stochastic differential equations. Commun. Math. Phys. 89, 13-17 (1983)

27. Davies, E.B.: Some contraction semigroups in quantum probability. Z. Wahrscheinlichkeitstheorie 23, 261-273 (1972)

28. Balslev, E., Manuceau, J., Verbeure, A.: Representations of anticommutation relations and Bogolioubov transformations. Commun. Math. Phys. 8, 315-326 (1968)

29. Rocca, F., Sirugue, M., Testard, D.: On a class of equilibrium states under the Kubo-MartinSchwinger boundary condition. I. Fermions. Commun. Math. Phys. 13, 317-334 (1969)

30. Sirugue, M., Winnink, M.: Constraints imposed upon a state of a system that satisfies the KMS boundary condition. Commun. Math. Phys. 19, 161-168 (1970)

31. Bratteli, O., Robinson, D.W.: Green functions, Hamiltonians and modular automorphisms. Commun. Math. Phys. 50, 133-156 (1976)

32. Carmichael, H.J., Walls, D.F.: Detailed balance in open quantum Markoffian systems. Z. Phys. B23, 299-306 (1976)

33. Frigerio, A., Gorini, V.: Diffusion processes, quantum dynamical semigroups, and the classical KMS condition. Preprint IFUM 276/FT, 1982. J. Math. Phys. (to appear)

Communicated by H. Araki

Received July 26, 1983; in revised form November 14, 1983 